Bull. Korean Math. Soc. 50 (2013), No. 5, pp. 1513-1521

http://dx.doi.org/10.4134/BKMS.2013.50.5.1513

\title{
CYCLIC CODES OVER SOME SPECIAL RINGS
}

\author{
Cristina Flaut
}

\begin{abstract}
In this paper we will study cyclic codes over some special rings: $\mathbb{F}_{q}[u] /\left(u^{i}\right), \mathbb{F}_{q}\left[u_{1}, \ldots, u_{i}\right] /\left(u_{1}^{2}, u_{2}^{2}, \ldots, u_{i}^{2}, u_{1} u_{2}-u_{2} u_{1}, \ldots, u_{k} u_{j}-\right.$ $\left.u_{j} u_{k}, \ldots\right)$, and $\mathbb{F}_{q}[u, v] /\left(u^{i}, v^{j}, u v-v u\right)$, where $\mathbb{F}_{q}$ is a field with $q$ elements $q=p^{r}$ for some prime number $p$ and $r \in \mathbb{N}-\{0\}$.
\end{abstract}

\section{Introduction}

Codes over finite rings have been intensively studied in the last time, some of the earliest results of them are in [5], [18]. Ones of the most important finite rings in the coding theory are: the finite field $\mathbb{F}_{q}$ and the ring $\mathbb{Z}_{q}$, where $q=p^{r}$ for some prime number $p$ and $r \in \mathbb{N}-\{0\}$. For example, in the paper [10] some codes over $\mathbb{Z}_{4}$ are investigated. The class of cyclic codes is an important class of linear codes with a big interest in coding theory. Described as ideals in certain polynomial rings, they have a good algebraic structure and the cyclic codes over some special finite rings were recently described (see [2], [3], [6], [9], [14], [15], [19]). Two classes of these main rings are: Galois rings and rings of the form $\mathbb{F}_{q}[u] /\left(u^{i}\right)$ or generalization of these, where $q=p^{r}$ for some prime number $p$ and $r \in \mathbb{N}-\{0\}$.

In this paper, we will investigate the structure of cyclic codes of arbitrary length over the rings:

$$
\begin{aligned}
& \mathbb{F}_{q}[u] /\left(u^{i}\right), \\
& \mathbb{F}_{q}\left[u_{1}, \ldots, u_{i}\right] /\left(u_{1}^{2}, u_{2}^{2}, \ldots, u_{i}^{2}, u_{1} u_{2}-u_{2} u_{1}, \ldots, u_{k} u_{j}-u_{j} u_{k}, \ldots\right), \\
& \mathbb{F}_{q}[u, v] /\left(u^{i}, v^{j}, u v-v u\right) .
\end{aligned}
$$

\section{Preliminaries}

The Galois ring $G R(q, n)$ is the residue class ring $\mathbb{Z} / p^{r} \mathbb{Z}[x] /(f(x))$, where $f(x)$ is a monic irreducible polynomial of degree $n$ in $\mathbb{Z}_{p^{r}}[x]$ such that $f(x) \bmod$ $p$ is a monic irreducible polynomial in $\mathbb{Z}_{p}[x]$. The existence of the polynomial $f(x)$ is given by the Hensel lifting, which allows us to "lift" a root $\rho$ of a

Received April 17, 2012; Revised November 15, 2012.

2010 Mathematics Subject Classification. 94B15, 94B05.

Key words and phrases. cyclic codes, codes over rings, Hamming distance.

(C)2013 The Korean Mathematical Society 
polynomial $f \bmod p^{t}$ to a new root $\sigma$ for the polynomial $f \bmod p^{t+1}, t \in \mathbb{N}-\{0\}$ (see [8], [13]). From here, it results that we can choose the polynomial $f$, monic and irreducible over $\mathbb{Z}_{p}$, as in the standard construction of the Galois field $\mathbb{F}_{p^{r}}$ from $\mathbb{Z}_{p}$, and we lift it to a polynomial over $\mathbb{Z} / p^{r} \mathbb{Z}$. We remark that $|G R(q, n)|=p^{r m}$. For example, $G R(q, 1)=\mathbb{Z}_{q}$ and $G R(p, r)=\mathbb{F}_{q}$. Let $\theta$ be a root of the polynomial $f(x)$. Since we can think at $G R(q, n)$ as a Galois extension $\mathbb{Z}_{p^{r}}[\theta]$ of $\mathbb{Z}_{p^{r}}$ by a root $\theta$ of $f(x)$, each element $v \in G R(q, n)$ has the form

$$
v=a_{0}+a_{1} \theta+a_{2} \theta^{2}+\cdots+a_{n-1} \theta^{n-1},
$$

where $a_{i} \in \mathbb{Z}_{p^{r}}, i \in\{0,1, \ldots, n-1\}$ (see [16] and [17]).

The Galois ring $G R(q, n)$ is a free module of rank $n$ over $\mathbb{Z}_{q}$ and the set

$$
\left\{1, \theta, \theta^{2}, \ldots, \theta^{n-1}\right\}
$$

is a free basis for $G R(q, n)$. Since the ring $\mathbb{Z}_{p^{r}}$ satisfies the invariant dimension property, it results that all bases in $G R(q, n)$ have $n$ elements.

Let $R$ be a commutative ring and $I$ be an ideal of the ring $R$. The ideal $I$ is called principal if it is generated by one element. The ring $R$ is called principal if all its ideals are principal. The ring $R$ is called a local ring if it has a unique maximal ideal. A ring $R$ is called a chain ring if the set of all ideals of $R$ is ordered by inclusion (is a chain under set inclusion). For a chain ring its unique maximal ideal contains the nilpotent elements.

All ideals in a finite chain ring $R$ are principal. Indeed, if $I$ is not a principal ideal, since $R$ is finite, we have that $I$ is finite generated and $I=\left\langle a_{1}, \ldots, a_{t}\right\rangle$, where $\left\{a_{1}, \ldots, a_{t}\right\}$ is a minimal set of generators. It results $\left\langle a_{i}\right\rangle \varsubsetneqq\left\langle a_{j}\right\rangle, i \neq$ $j, i, j \in\{1,2\}$, which is a contradiction, since $R$ is a chain ring. We obtain that all ideals in a finite chain ring are principal and there is a unique maximal ideal. It results that a chain ring is a local ring. For details about the chain rings, the reader is referred to [12].

Let $\boldsymbol{m}$ be the maximal ideal in a finite chain ring and let $u$ be its generator, i.e., $\boldsymbol{m}=\langle u\rangle=R u$. Since $R$ is finite, the chain $R=\left\langle u^{0}\right\rangle \supseteq\left\langle u^{1}\right\rangle \supseteq\left\langle u^{2}\right\rangle \supseteq$ $\cdots\left\langle u^{j}\right\rangle \supseteq \cdots$ is a finite chain. It results that there is an element $j$ with the property $\left\langle u^{j}\right\rangle=0$. The smallest number $t$ such that $\left\langle u^{t}\right\rangle=0$ is called the nilpotency index of $u$. The residue field $\mathbb{F}=R / \boldsymbol{m}$ has $q=p^{t}$ elements with $p$ a prime number, char $\mathbb{F}=p$ and $\left|\mathbb{F}^{*}\right|=p^{t}-1$.

For details about the finite chain rings, the reader is referred to [12].

Galois rings or the rings of the form $\mathbb{F}_{q}[u] /\left(u^{i}\right)$ are principal ideal rings.

Galois ring $G R\left(p^{r}, n\right)$ is a finite chain ring (of length $r$ ).

Finite chain rings allow us to find good description for cyclic codes over these rings.

Let $R$ be a unitary finite commutative ring. A code $C$ of length $n$ over $R$ is a nonempty subset of $R^{n}=\underbrace{R \times R \times \cdots \times R}_{n \text {-times }}$. The elements of $C$ are called codewords. A linear code $C$ of length $n$ over $R$ is a $R$-submodule of $R^{n}$. We remark that such a submodule is not necessary a free module. A linear code 
$C$ of length $n$ is a cyclic code if for each codeword $c=\left(c_{0}, \ldots, c_{n-1}\right) \in C$, the codeword $\left(c_{n-1}, c_{0}, \ldots, c_{n-2}\right)$ belongs to $C$.

For the cyclic codes, we will write the codewords as polynomials. Let $C$ be a cyclic code. For each $c=\left(c_{0}, \ldots, c_{n-1}\right) \in C$ we associate the polynomial $c(x)$ of degree less than $n, c(x)=c_{0}+c_{1} x+\cdots+c_{i} x^{i}+\cdots+c_{n-1} x^{n-1} \in R[x]$, called the associated polynomial. The codeword $\bar{c}=\left(c_{n-1}, c_{0}, \ldots, c_{n-2}\right)$ has the associated polynomial $\bar{c}(x)=c_{n-1}+c_{0} x+\cdots+c_{i} x^{i+1}+\cdots+c_{n-2} x^{n-1}$ and we have $\bar{c}(x)=c(x) x-c_{n-1}\left(x^{n}-1\right)$, therefore $\bar{c}(x)=c(x) x \bmod \left(x^{n}-1\right)$. We remark that $c(x) \in C \bmod \left(x^{n}-1\right)$ if and only if $c(x) x \in C \bmod \left(x^{n}-1\right)$. Using induction steps, $c(x) x \in C \bmod \left(x^{n}-1\right)$ if and only if $c(x) x^{2} \in C \bmod$ $\left(x^{n}-1\right)$. Therefore we have $c(x) x^{i} \in C \bmod \left(x^{n}-1\right)$ for all $i \in \mathbb{N}-\{0\}$. From here, it results that $C$ is a cyclic code of length $n$ over $R$ if and only if $C$ is an ideal in the ring $R[x] /\left(x^{n}-1\right)$.

\section{The rings}

With the above notations, we consider the rings

$$
\begin{gathered}
R_{i} \simeq \mathbb{F}_{q}[u] /\left(u^{i}\right), \\
S_{i} \simeq \mathbb{F}_{q}\left[u_{1}, \ldots, u_{i}\right] /\left(u_{1}^{2}, u_{2}^{2}, \ldots, u_{i}^{2}, u_{1} u_{2}-u_{2} u_{1}, \ldots, u_{k} u_{j}-u_{j} u_{k}, \ldots\right), k \neq j, \\
T_{(i, j)}=\mathbb{F}_{q}[u, v] /\left(u^{i}, v^{j}, u v-v u\right), i, j \in \mathbb{N}-\{0\} .
\end{gathered}
$$

For example, the ring $R_{i}$ is a commutative chain ring and $\langle u\rangle$ is a maximal ideal (see [7]).

For $R \in\left\{R_{i}, S_{i}, T_{(i, j)}\right\}, i, j, n \in \mathbb{N}-\{0\}$, we denote

$$
\begin{aligned}
R_{i, n} & =R_{i}[x] /\left(x^{n}-1\right), \\
S_{i, n} & =S_{i}[x] /\left(x^{n}-1\right), \\
T_{(i, j), n} & =T_{(i, j)}[x] /\left(x^{n}-1\right) .
\end{aligned}
$$

Remark 2.1. Since the rings $R_{i}, S_{i}, T_{(i, j)}$ are finite rings, the rings $R_{i, n}$, $S_{i, n}, T_{(i, j), n}$ are isomorphic with the group ring $R G$, where $G=\left(g / g^{n}-1=0\right)$ is the cyclic group of order $n$ and $R \in\left\{R_{i, n}, S_{i, n}, T_{(i, j), n}\right\}$.

Remark 2.2. If the characteristic of the ring is not prime with the length $n$ of the code, then the polynomial $x^{n}-1$ factors uniquely over $\mathbb{F}_{q}$, but does not factor uniquely over the rings $R_{i}, S_{i}, T_{(i, j)}$. Indeed, for example, for $p=2, r=$ $2, i=3, j=2, n=2$, we have $x^{2}-1=(x-1)^{2}=\left(x-\left(1-u^{2}\right)\right)^{2}$ over $R_{3}$, $x^{2}-1=(x-1)^{2}=\left(x-\left(1+u_{1}^{2}+u_{2}^{2}+u_{3}^{2}\right)\right)^{2}$ over $S_{3}, x^{2}-1=(x-1)^{2}=$ $\left(x-\left(1+u^{2}+v\right)\right)^{2}$ over $T_{(3,2)}$.

In [15], if $\operatorname{gcd}(n, p)=1$, the authors proved that $R_{i, n}$ is a principal ideal ring. In the case of the rings $S_{i, n}$ and $T_{(i, j), n}$ situation is not the same.

Proposition 2.3. The rings $S_{i, n}$ and $T_{(i, j), n}$ are not principal ideal rings. 
Proof. In the following, we will use some ideas given in [19], Lemma 2.4, when the authors proved the above result for $S_{i, n}$ in the case when $i=2, p=2, r=1$.

Let $R \in\left\{S_{i}, T_{(i, j)}\right\}$. From Remark 2.1, we define the ring morphism $\varphi$ : $R G \rightarrow R, \varphi\left(c_{0}+c_{1} x+\cdots+c_{n-1} x^{n-1}\right)=c_{0}+c_{1}+\cdots+c_{n-1}$, which is a surjective map, called the augmentation morphism. Let $I_{i}=\left(u_{1}, \ldots, u_{i}\right)$ be the ideal in $S_{i}$ generated by the elements $\left\{u_{1}, \ldots, u_{i}\right\}$ and $I_{u, v}$ be the ideal in $T_{(i, j)}$ generated by the elements $\{u, v\}$. These ideals are not principal ideals. Let $I \in\left\{I_{i}, I_{u, v}\right\}$. We have that $\varphi^{-1}(I)=J$ is an ideal in $R G$. Since $\varphi$ is surjective, therefore $\varphi(J)$ is an ideal in $R$ and $\varphi(J)=\varphi\left(\varphi^{-1}(I)\right)=I$. From here, if $J$ is a principal ideal, it results that $I$ is a principal ideal, false.

Proposition 2.4. With the above notations, for $n=p^{l} k$, with $k>1, \operatorname{gcd}(p, k)$ $=1$, the rings $R_{i, n}, S_{i, n}$ and $T_{(i, j), n}$ are not local rings.

Proof. From [11], we know that a ring is local if and only if the non-units form a maximal ideal in the ring. Let $R \in\left\{R_{i, n}, S_{i, n}, T_{(i, j), n}\right\}$.

Case $1 . \operatorname{gcd}(p, k-1) \neq 1$. From the hypothesis, in $R$, we have

$$
0=x^{p^{l} k}-1=\left(x^{p^{l}}-1\right)\left(x^{p^{l}(k-1)}+x^{p^{l}(k-2)}+\cdots+1\right) .
$$

It results that $f(x)=x^{p^{l}(k-1)}+x^{p^{l}(k-2)}+\cdots+1$ is a zero divisor, so that it is not invertible in $R$. We obtain

$$
\varphi\left(x^{p^{l}(k-2)}+x^{p^{l}(k-2)}+\cdots+1\right)=\underbrace{1+1+\cdots+1}_{(k-1) \text {-times }}=0 .
$$

Then $g(x)=x^{p^{l}(k-2)}+x^{p^{l}(k-2)}+\cdots+1$ is a non-unit in $R$. Since $x^{p^{l}(k-1)} x^{p^{l}}=$ 1 , we have that $f(x)-g(x)=x^{p^{l}(k-1)}$ is a unit in $R$. Therefore non-invertible elements in $R$ do not form an ideal, hence $R$ is not a local ring.

Case 2. $\operatorname{gcd}(p, k-1)=1$. Let $g_{1}=u g, g_{1}$ be a non-unit element. Such an element is a nilpotent element. Denoting $h(x)=f(x)+g_{1}(x)$, we have $\varphi(h(x))=k+(k-1) u$. Since $\operatorname{gcd}(p, k-1)=1$, it results that $(k-1) u$ is a nonzero nilpotent element. From here, we obtain that $k+(k-1) u$ is a sum between an invertible element, $k$, and a nilpotent element, therefore it is an invertible element. It results that $\varphi(h(x))$ is a unit and $h(x)$ is also a unit, hence non-invertible elements do not form an ideal. We just proved that $R$ is not a local ring.

Proposition 2.5. With the above notations, for $n=p^{l}$ the rings $R_{i, n}, S_{i, n}$, $T_{(i, j), n}$ are local rings.

Proof. Let $R_{n} \in\left\{R_{i, n}, S_{i, n}, T_{(i, j), n}\right\}, R \in\left\{R_{i}, S_{i}, T_{(i, j)}\right\}$. We will prove that the non-units form an ideal in the ring $R_{n}$. First of all, we remark that a non-unit element in $R_{n}$ different from zero is a zero divisor. Indeed, if $\alpha \in R_{n}, \alpha \neq 0$, is a non-invertible element, then the ideal generated by $\alpha$ is different from $R_{n}$. Hence we can find the elements $\alpha_{1} \neq \alpha_{2}$ such that $\alpha_{1} \alpha=\alpha_{2} \alpha$. Therefore $\left(\alpha_{1}-\alpha_{2}\right) \alpha=0$ and $\alpha$ is a zero divisor. We remark that an element $\theta$ in $R$ 
has the form $\theta=\theta_{1}+\theta_{2}$, where $\theta_{1} \in \mathbb{F}_{q}$ and $\theta_{2} \in R-\mathbb{F}_{q}$. If $\theta$ is a unit in $R$, then $\theta_{1} \in \mathbb{F}_{q}^{*}$. Let $s=s_{0}+s_{1} x+\cdots+s_{n-1} x^{n-1} \in R_{n}$ and $s_{k}=\left(s_{k}\right)_{1}+\left(s_{k}\right)_{2}$, where $\left(s_{k}\right)_{1} \in \mathbb{F}_{q}$ and $\left(s_{k}\right)_{2} \in R-\mathbb{F}_{q}, j \in\{1, \ldots, n-1\}$. Since $x^{p^{j}}=1$, it results that $s^{p^{j}}=s_{0}^{p^{j}}+s_{1}^{p^{j}}+\cdots+s_{n-1}^{p^{j}}$ and $s^{p^{j}}$ is a unit or a zero divisor. If $s^{p^{j}}$ is a zero divisor, then $s$ is a zero divisor, hence a non-unit. If $s^{p^{j}}$ is a unit, it results that $s$ is a unit, since $s \cdot s^{p^{j}-1}=s^{p^{j}}$. If $s^{p^{j}}$ is a zero divisor, then $\sum_{k=1}^{n-1}\left(s_{k}^{p^{j}}\right)_{1}=0$ and this characterize a zero-divisor, hence a non-unit in the ring $R_{n}$. Let $t=t_{0}+t_{1} x+\cdots+t_{n-1} x^{n-1} \in R_{n}$, with $\sum_{k=1}^{n-1}\left(t_{k}^{p^{j}}\right)_{1}=0$, be another non-unit. The element $r=s+t$ is also a non-unit. For prove this, we compute $\sum_{k=1}^{n-1}\left(r_{k}^{p^{j}}\right)_{1}$. It results $\sum_{k=1}^{n-1}\left(r_{k}^{p^{j}}\right)_{1}=\sum_{k=1}^{n-1}\left(s_{k}+t_{k}\right)_{1}^{p^{j}}=$ $\sum_{k=1}^{n-1}\left(s_{k}^{p^{j}}\right)_{1}+\sum_{k=1}^{n-1}\left(t_{k}^{p^{j}}\right)_{1}=0+0=0$, therefore $r$ is a non-unit and non-units form an ideal. Hence $R$ is a local ring.

Propositions 2.5 and 2.6 were proved for the ring $S_{i, n}$ in [19] in the particular case $i=2$ (see Theorem 2.5 and Theorem 2.7).

\section{Ranks for the cyclic codes over the rings $R_{i}, S_{i}, T_{(i, j)}$}

In [4], Proposition 1, the authors described cyclic codes of length $n$ over the Galois ring $G R(q, l)=\mathbb{Z}_{p^{m}}[x] /(f), \operatorname{deg} f=l, q=p^{m},(n, q)=1$. Using some ideas given in this proof, we can describe cyclic codes over the rings $R_{i}, S_{i}, T_{(i, j)}$ in the general case.

Proposition 3.1. Let $R \in\left\{R_{i}, S_{i}, T_{(i, j)}\right\}$. A non-zero cyclic code $C$ of length $n$ over $R$ is a free module over $R$ if it is generated by a monic polynomial $h(x)$, where $h(x) \mid\left(x^{n}-1\right)$ over $R$. In this case, rank $C=n-r, \operatorname{deg} h(x)=r$, and $\left\{h(x), x h(x), \ldots, x^{n-r-1} h(x)\right\}$ is a basis in $C$.

Proof. Let $C$ be a non-zero cyclic code $C$ of length $n$ over $R$ generated by the polynomial $h(x)$, where $h(x) \mid\left(x^{n}-1\right)$. Since $h(x) \mid\left(x^{n}-1\right)$, we can consider $h(x)$ a monic polynomial. Then there is a monic polynomial $q(x) \in R$ such that $h(x) q(x)=x^{n}-1$. From here, it results that $\operatorname{deg} q(x)=n-r$ and $q$ has the form $q(x)=q_{0}+q_{1} x+\cdots+x^{n-r}$. Let $R_{n} \in\left\{R_{i, n}, S_{i, n}, T_{(i, j), n}\right\}$. In $R_{n}$ we have $h(x) q(x)=h(x)\left(q_{0}+q_{1} x+\cdots+x^{n-r}\right)=0$. Therefore $x^{i} h(x)$, for $i \geq$ $n-r$, can be written as a linear combination of the elements $\{h(x), x h(x), \ldots$, $\left.x^{n-r} h(x)\right\}$, hence each element in $C$ of the form $p(x) h(x), p(x) \in R_{n}$ is a linear combination of $\left\{h(x), x h(x), \ldots, x^{n-r-1} h(x)\right\}$. It results that the system $\left\{h(x), x h(x), \ldots, x^{n-r-1} h(x)\right\}$ spans $C$. For linearly independence over $R$, let $\alpha_{0}, \ldots, \alpha_{n-r-1} \in R$ such that $\alpha_{0} h(x)+\alpha_{1} x h(x)+\cdots+\alpha_{n-r-1} x^{n-r-1} h(x)=0$. We obtain $\left(\alpha_{0}+\alpha_{1} x+\cdots+\alpha_{n-r-1} x^{n-r-1}\right) h(x)=0$ in $R_{n}$, hence $\left(x^{n}-1\right)$ $\left(\alpha_{0}+\alpha_{1} x+\cdots+\alpha_{n-r-1} x^{n-r-1}\right) h(x)$ in $R[x]$, with

$$
\operatorname{deg}\left(\alpha_{0}+\alpha_{1} x+\cdots+\alpha_{n-r-1} x^{n-r-1}\right) h(x)=n-1<n .
$$


From here, we have $\left(\alpha_{0}+\alpha_{1} x+\cdots+\alpha_{n-r-1} x^{n-r-1}\right) h(x)=0$ in $R[x]$. Since $h(x)$ is monic, it results $\alpha_{0}+\alpha_{1} x+\cdots+\alpha_{n-r-1} x^{n-r-1}=0$, hence $\alpha_{0}=\alpha_{1}=$ $\cdots=\alpha_{n-r-1}=0$.

We remark that another proof of the above results can be obtained using the main result from [9].

Corollary 3.2. With the notations used in Proposition 3.1, if $C$ is a nonzerocyclic code of length $n$ over $R$ generated by a monic polynomial $h(x)$, where $h(x) \mid\left(x^{n}-1\right)$ over $R$, then $|C|=|R|^{n-r}$.

Proposition 3.3. Let $C$ be a non-zero cyclic code of length $n$ over $R \in$ $\left\{R_{i}, S_{i}, T_{(i, j)}\right\}$ generated by the polynomials $\left\{h_{1}, \ldots, h_{t}\right\}$. Therefore $C$ is a vector space over $\mathbb{F}_{q}$ and $|C| \leq|q|^{s n}$, where $s=\operatorname{dim}_{\mathbb{F}_{q}} R$.

Proof. We know that $R$ is a vector space over $\mathbb{F}_{q}$. Let $s=\operatorname{dim}_{\mathbb{F}_{q}} R$ and $\left\{1, v_{1}, \ldots, v_{s-1}\right\}$ be a basis in $R$. We will prove that $B=\left\{1, x, \ldots, x^{n-1}, v_{1}, v_{1} x\right.$, $\left.\ldots, v_{1} x^{n-1}, \ldots, v_{s-1}, v_{s-1} x, \ldots, v_{s-1} x^{n-1}\right\}$ is a basis in the $\mathbb{F}_{q \text {-vector space }}$ $R_{n}, R_{n} \in\left\{R_{i, n}, S_{i, n}, T_{(i, j), n}\right\}$.

First, we will show the linearly independence of the elements from $B$. If there are the elements $\alpha_{1, i_{1}}, \alpha_{2 i_{2}}, \ldots, \alpha_{s i_{s}} \in \mathbb{F}_{q}, i_{j} \in\{0,1,2, \ldots, n-1\}, j \in$ $\{1,2, \ldots, s\}$ such that $\alpha_{1,0} \cdot 1+\cdots+\alpha_{1, n-1} x^{n-1}+\cdots+\alpha_{s, 0} v_{s-1}+\cdots+$ $\alpha_{s, n-1} v_{s-1} x^{n-1}=0$, comparing the coefficients in this equation, we get

$$
\alpha_{1,0} \cdot 1+\alpha_{2,0} v_{s-1}+\cdots+\alpha_{s, 0} v_{s-1}=0 .
$$

Since $v_{1}, \ldots, v_{s-1}$ are nilpotent elements in $R$, if $\alpha_{1,0} \neq 0$, from relation (3.1), we obtain that a unit is equal with a nilpotent element, false. Hence $\alpha_{1,0}=0$ and $\alpha_{2,0} v_{s-1}+\cdots+\alpha_{s, 0} v_{s-1}=0$, therefore $\alpha_{2,0}=\cdots=\alpha_{s, 0}=0$. In the same way, comparing coefficients of $x, x^{2}, \ldots, x^{n-1}$ with zero, we have $\alpha_{1, i_{1}}=\alpha_{2, i_{2}}=\cdots=\alpha_{s, i_{s}}=0$ for all $i_{j} \in\{0,1,2, \ldots, n-1\}, j \in\{1,2, \ldots, s\}$.

We will prove that $B$ generates $R_{n}$. Let $f(x) \in R_{n}$. By straightforward calculations, we obtain that $f(x)$ is a linear combination of elements in $B$ with coefficients in $\mathbb{F}_{q}$. It results $\left|R_{n}\right|=|q|^{s n}$.

Now, let $C$ be a nonzero cyclic code. Then $C$ is a vector subspace of the $\mathbb{F}_{q}$-vector space $R_{n}$, therefore $|C| \leq|q|^{\text {sn }}$.

In [2], Theorem 3, and in [3], Theorem 4.2, the authors gave a basis or a minimal spanning set for the codes of even length over $\mathbb{Z}_{2}+u \mathbb{Z}_{2}$, respectively $\mathbb{Z}_{2}+u \mathbb{Z}_{2}+\cdots+u^{k-1} \mathbb{Z}_{2}$. The same description could be done, in general case, over the ring $R_{i}$. If we have supplementary relations between polynomials $h_{1}, \ldots, h_{t}$, we can compute $|C|$, as we can see in the following examples.

Example 3.4. Over $R_{i}$, for $i=2$, using Theorem 3 from [2], if $C$ is a nonzero cyclic code of length $n,(n, p) \neq 1$, and $C=(g(x)+u p(x), u a(x))$, $a(x)|g(x)|\left(x^{n}-1\right)$, with $\operatorname{deg} g(x)=r, \operatorname{deg} a(x)=t, r \geq t, \operatorname{deg} a(x)>$ $\operatorname{deg} p(x)$, then $|C|=(q)^{2 n-r-t}$. Indeed, $C$ is a vector space over $\mathbb{F}_{q}$ and let 
$B=\left\{g(x)+u p(x), x(g(x)+u p(x)), \ldots, x^{n-r-1}(g(x)+u p(x)), u a(x), x u a(x), \ldots\right.$, $\left.x^{n-t-1} u a(x)\right\}$. We will prove that $B$ is a basis in the $\mathbb{F}_{q}$-vector space $C$.

First, we will show that $B$ spans $C$. Let $c(x) \in C$. Then

$$
c(x)=q_{1}(x)(g(x)+u p(x))+q_{2}(x) u a(x), q_{i}(x) \in R[x], i \in\{1,2\} .
$$

If $\operatorname{deg} q_{1}(x)<n-r$ and $\operatorname{deg} q_{2}(x)<n-t$, we have $B$ spans $C$. If $\operatorname{deg} q_{1}(x) \geq$ $n-r$ or $\operatorname{deg} q_{2}(x) \geq n-t$, it suffices to show that $x^{n-r}(g(x)+u p(x))$, $u(g(x)+u p(x))$ and $x^{n-t} u a(x)$ are generated by $B$ over $\mathbb{F}_{q}$.

We have $x^{n-r}(g(x)+u p(x))=x^{n}-1+q(x), \operatorname{deg} q(x) \leq n-1$. But $q(x) \in C$ and, by the division algorithm, we have $q(x)=(g(x)+u p(x)) h_{1}(x)+s_{1}(x)$, $\operatorname{deg} s_{1}(x)<r, \operatorname{deg} h_{1}(x) \leq n-1, s_{1}(x)=u a(x) h_{2}(x)+s_{2}(x), \operatorname{deg} s_{2}(x)<$ $\operatorname{deg} u a(x), \operatorname{deg} h_{2}(x) \leq r-t$. Since $\operatorname{deg} u a(x)=\operatorname{deg} a(x)$ and $i n C$ any polynomial must have degree greater or equal with $\operatorname{deg} a(x)$, it results $s_{2}(x)=0$. Since $a(x) \mid g(x)$, hence $g(x)=a(x) h(x)$ and we have $u g(x)=u(g(x)+u p(x))=$ $u a(x) h(x)$, with $\operatorname{deg} h(x) \leq r-t<n-t-1$. It results that $u(g(x)+u p(x))$ is generated by $B$ over $\mathbb{F}_{q}$. To finish the proof, it is enough to show that the element $u x^{r-t} a(x)$ is generated by $B$ over $\mathbb{F}_{q}$. We have $u x^{r-t} a(x)=$ $u(g(x)+u p(x))+u h_{3}(x)$, where $u h_{3}$ belongs to $C$ and $t \leq \operatorname{deg} h_{3}(x)<r$. Therefore $u h_{3}(x)=\alpha_{0} u a(x)+\alpha_{1} x u a(x)+\cdots+\alpha_{r-t-1} x^{r-t-1} u a(x), \alpha_{i} \in \mathbb{F}_{q}, i \in$ $\{0, \ldots, r-t-1\}$.

We will prove that $B$ is a linearly independent system. Indeed, if there are the elements $\alpha_{1, i_{1}}, \alpha_{2, i_{2}} \in \mathbb{F}_{q}, i_{j} \in\{0,1,2, \ldots, n-1\}, j \in\{1,2\}$ such that $\alpha_{1,0}(g(x)+u p(x))+\cdots+\alpha_{1, n-1} x^{n-r-1}(g(x)+u p(x))+\alpha_{2,0} u a(x)+\cdots+$ $\alpha_{2, n-1} x^{n-t-1} u a(x)=0$, comparing the coefficients in this equation, we get $\alpha_{1,0} g(0)+\alpha_{1,0} u p(0)+\alpha_{2,0} u a(0)=0$. If $\alpha_{1,0} \neq 0$, since $g(0)$ is a unit and $u$ a nilpotent element, it results that a unit is equal with a nilpotent, false. Therefore $\alpha_{1,0}=0$. We obtain $\alpha_{2,0} u a(0)=0$. If $\alpha_{2,0} \neq 0$, it results $u a(0)=$ 0 , false, since $a(0)$ is a unit. We repeat this procedure and we get $\alpha_{1, i_{1}}=$ $\alpha_{2, i_{2}}=0$ for all $i_{j} \in\{0,1,2, \ldots, n-1\}, j \in\{1,2\}$, hence $B$ is a linearly independent system. It results that $B$ is a basis in the $\mathbb{F}_{q}$-vector space $C$ and $|C|=|q|^{2 n-r-t}$.

\section{Minimum Hamming distance for the cyclic codes over the rings$$
R_{i}, S_{i}, T_{(i, j)}
$$

Let $C$ be a linear code over the ring $R$. The Hamming distance between two codewords $c_{1}$ and $c_{2}$, denoted by $H\left(c_{1}, c_{2}\right)$, is the number of coordinates in which the codewords $c_{1}$ and $c_{2}$ differ. The number of nonzero entries of a codeword $c$, denoted $w(c)$, is called the Hamming weight of the codeword $c$. The Hamming distance of a linear code $C$ is

$$
d(C)=\min \{w(c) \mid c \in C, c \neq 0\} .
$$

In [1], the authors studied the Hamming distance of cyclic codes of even length, especially codes of length $2^{e}, e \in \mathbb{N}-\{0\}$ (Lemmas 16, 17, and 18). In the following, using some ideas from the mentioned lemmas, we will investigate the 
Hamming distance for cyclic codes of length $n=p^{r}, r \in \mathbb{N}-\{0\}$, over the rings $R_{i}, S_{i}, T_{(i, j)}$.

Definition 4.1. Let $n=a_{s-1} p^{s-1}+a_{s-2} p^{s-2}+\cdots+a_{1} p^{1}+a_{0} p^{0}, \alpha_{i} \in$ $\{0,1, \ldots, p-1\}, i \in\{0,1, \ldots, s-1\}$, be the $p$-adic expansion of $n$.

1) If $a_{s-1}=\cdots=a_{s-t} \neq 0, s-t>0$ and $a_{s-i}=0$ for all $i \in\{t+2, t+$ $3, \ldots, s-1\}$, then $n$ has a $p$-adic length $t$ zero expansion.

2) If $a_{s-1}=\cdots=a_{s-t} \neq 0, s-t>0$ and $a_{s-i} \neq 0$ for some elements $i \in\{t+2, t+3, \ldots, s-1\}$, then $n$ has a $p$-adic length $t$ non-zero expansion.

3 ) If $s=t$, then $n$ has a $p$-adic full expansion.

Proposition 4.2. Let $C=(g(x))$ be a cyclic code over $R \in\left\{R_{i}, S_{i}, T_{(i, j)}\right\}$ of length $p^{r}, r \in \mathbb{N}-\{0\}$, where $g(x)=\left(x^{a p^{r-1}}-1\right) g_{1}(x)$. If $g_{1}(x)$ generates a cyclic code of length $p^{r-1}$ and Hamming distance $d$, then $d(C)=2 d$.

Proof. For $c \in C$ we have $c=\left(x^{a p^{r-1}}-1\right) g_{1}(x) g_{2}(x), g_{2}(x) \in \mathbb{F}_{q}[x] /\left(x^{n}-\right.$ 1) and $g_{1}(x) g_{2}(x) \in\left(g_{1}(x)\right)$. It results $w(c)=w\left(\left(x^{a p^{r-1}}-1\right) g_{1}(x) g_{2}(x)\right)=$ $w\left(x^{a p^{r-1}} g_{1}(x) g_{2}(x)\right)+w\left(g_{1}(x) g_{2}(x)\right)$. Then $d(C)=d+d=2 d$.

Conclusion. In this paper we investigate the structure of cyclic codes of arbitrary length over the rings $R_{i}, S_{i}, T_{(i, j)}$. Moreover the ranks and minimum Hamming distance of these codes were studied. Since the rings with Hamming weight cannot produce always better codes, a more relevant weight as, for example, the homogeneous weight on the above mentioned rings can be studied. The remark above can constitute the starting point for further research.

Acknowledgements. I would like to thank the referee for his/her many suggestions which helped me improve this paper.

\section{References}

[1] T. Abualrub and I. Siap, On the construction of cyclic codes over the ring $\mathbb{Z}_{2}+u \mathbb{Z}_{2}$, WSEAS Trans. Math. 5 (2006), no. 6, 750-755.

[2] Cyclic codes over the rings $\mathbb{Z}_{2}+u \mathbb{Z}_{2}$ and $\mathbb{Z}_{2}+u \mathbb{Z}_{2}+u^{2} \mathbb{Z}_{2}$, Des Codes Cryptogr. 42 (2007), no. 3, 273-287.

[3] M. M. Al-Ashker and M. Hamoudeh, Cyclic codes over $\mathbb{Z}_{2}+u \mathbb{Z}_{2}+\cdots+u^{k-1} \mathbb{Z}_{2}$, Turk J. Math. 34 (2010), 1-13.

[4] M. Bhaintwal and S. K. Wasan, On quasi-cyclic codes over $\mathbb{Z}_{q}$, Appl. Algebra Engrg. Comm. Comput. 20 (2009), no. 5-6, 459-480.

[5] I. F. Blake, Codes over certain rings, Inf. Control 20 (1972), 396-404.

[6] S. T. Dougherty, S. Karadeniz, and B. Yildiz, Cyclic codes over $R_{k}$, Des. Codes Cryptogr. 63 (2012), no. 1, 113-126.

[7] S. T. Dougherty, H. Liu, and Y. H. Park, Lifted codes over finite chain rings, Math. J. Okayama Univ. 53 (2011), 39-53.

[8] D. Eisenbud, Commutative Algebra, Graduate Texts in Mathematics, 150, SpringerVerlag, Berlin, New York, 1995.

[9] M. Greferath, Cyclic codes over finite rings, Discrete Math. 177 (1997), no. 1-3, 273277. 
[10] A. R. Hammons Jr., P. V. Kumar, A. R. Calderbank, N. J. A. Sloane, and P. Solé, The $Z_{4}$ liniarity of Kerdock, Preparata, Goethals and related codes, IEEE Trans. Inform. Theory 40 (1994), no. 2, 301-319.

[11] T. W. Hungerford, Algebra, Springer Verlag, New York, 1974.

[12] B. R. McDonald, Finite Rings with Identity, New York, Marcel Dekker Inc., 1974.

[13] J. G. Milne, Étale cohomology, Princeton University Press, 1980.

[14] A. A. Nechaev and T. Honold, Fully weighted modules and representations of codes, (Russian) Problemy Peredachi Informatsii 35 (1999), no. 3, 18-39; translation in Problems Inform. Transmission 35 (1999), no. 3, 205-223.

[15] J.-F. Qian, L.-N. Zhang, and A.-X. Zhu, Cyclic codes over $\mathbb{F}_{p}+u \mathbb{F}_{p}+\cdots+u^{k-1} \mathbb{F}_{p}$, IEICE Trans. Fundamentals Vol. E88-A (2005), no. 3, 795-797.

[16] P. Solé and V. Sison, Bounds on the minimum homogeneous distance of the $p^{r}$-ary image of linear block codes over the Galois ring $G R\left(p^{r}, m\right)$, IEEE Trans. Inform. Theory $\mathbf{5 3}$ (2007), no. 6, 2270-2273.

[17] Quaternary convolutional codes from linear block codes over Galois rings, IEEE Trans. Inform. Theory 53 (2007), no. 6, 2267-2270.

[18] E. Spiegel, Codes over $Z_{m}$ revisited, Inform. and Control 37 (1978), no. 1, 100-104.

[19] B. Yildiz and S. Karadeniz, Cyclic codes over $\mathbb{F}_{2}+u \mathbb{F}_{2}+v \mathbb{F}_{2}+u v \mathbb{F}_{2}$, Des. Codes Cryptogr. 58 (2011), no. 3, 221-234.

Faculty of Mathematics and Computer Science

OVIDIUS UNIVERSITY

BD. Mamaia 124, 900527, Constanta, Romania

E-mail address: cflaut@univ-ovidius.ro; cristina_flaut@yahoo.com 